\title{
Reflets
}

Revue d'intervention sociale et communautaire

\section{Judiciarisation de la pauvreté et non-accès aux services juridiques : quand Kafka rencontre Goliath}

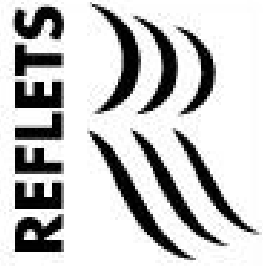

\section{Emmanuelle Bernheim}

Volume 25, numéro 1, printemps 2019

Actualité de la pauvreté : débats théoriques, défis pratiques

URI : https://id.erudit.org/iderudit/1064668ar

DOI : https://doi.org/10.7202/1064668ar

Aller au sommaire du numéro

Éditeur(s)

Reflets, Revue d'intervention sociale et communautaire

ISSN

1203-4576 (imprimé)

1712-8498 (numérique)

Découvrir la revue

Citer cet article

Bernheim, E. (2019). Judiciarisation de la pauvreté et non-accès aux services juridiques : quand Kafka rencontre Goliath. Reflets, 25(1), 71-93.

https://doi.org/10.7202/1064668ar
Résumé de l'article

La néolibéralisation du droit se traduit par le recours accru aux procédures juridiques associées au droit social pour sanctionner ou contrôler des personnes vivant en situation de pauvreté. C'est du moins ce que révèle l'étude des pratiques judiciaires québécoises en matière de logement, de santé mentale et de protection de la jeunesse. Parallèlement, depuis une vingtaine d'années, l'accessibilité des services juridiques n'a fait que diminuer, forçant un nombre de plus en plus important de personnes à faire face à la justice sans avocate ou avocat ou représentées par des avocates ou avocats sous-payés et débordés.
Tous droits réservés (C) Reflets, Revue d'intervention sociale et communautaire, 2019
Ce document est protégé par la loi sur le droit d'auteur. L'utilisation des services d'Érudit (y compris la reproduction) est assujettie à sa politique d'utilisation que vous pouvez consulter en ligne. 


\title{
Judiciarisation de la pauvreté et non-accès aux services juridiques : quand Kafka rencontre Goliath
}

\author{
Emmanuelle Bernheim \\ Professeure au département des sciences juridiques \\ Université du Québec à Montréal \\ Chercheuse au Centre de recherche de Montréal sur les inégalités sociales (CREMIS)
}

\section{Résumé}

La néolibéralisation du droit se traduit par le recours accru aux procédures juridiques associées au droit social pour sanctionner ou contrôler des personnes vivant en situation de pauvreté. C'est du moins ce que révèle l'étude des pratiques judiciaires québécoises en matière de logement, de santé mentale et de protection de la jeunesse. Parallèlement, depuis une vingtaine d'années, l'accessibilité des services juridiques n'a fait que diminuer, forçant un nombre de plus en plus important de personnes à faire face à la justice sans avocate ou avocat ou représentées par des avocates ou avocats sous-payés et débordés.

Mots-clés : droit néolibéral; judiciarisation de la pauvreté; logement; santé mentale; protection de la jeunesse; services juridiques; accès à la justice; non-représentation.

\section{Abstract}

The study of Quebec judicial practices in housing, mental health and youth protection reveal the increased use of legal procedures associated with social law to punish or control people living in poverty which is the proper of neoliberal law. At the same time, the accessibility of legal services has only diminished for the last twenty years, forcing a growing number of people to face justice without a lawyer or represented by underpaid and overworked lawyers.

Keywords : neoliberal law; judicialization of poverty; housing; mental health; youth protection; legal services; access to justice; unrepresented litigants. 


\section{Introduction}

La judiciarisation de la pauvreté, et plus particulièrement sa criminalisation, fait l'objet de recherches depuis des décennies : surreprésentation devant les tribunaux criminels et pénaux, nature des pratiques policières et judiciaires et adaptation des services judiciaires ont ainsi été largement documentées (Galanter, 1983; Laberge et Robert, 1992; Laberge et Morin, 1993; Foscarinis, 1996) et continuent de l'être aujourd'hui (Bellot et Sylvestre, 2017). Ces constats sont cohérents avec les travaux de référence sur le droit pénal en tant qu'outil de contrôle social, notamment ceux de Becker (1963), de Foucault (1975) et de Black (1983). Il appert qu'aujourd'hui, la judiciarisation de la pauvreté passe également par des canaux juridiques jusque-là réservés à des enjeux sociaux et l'étude de certaines pratiques judiciaires administratives et civiles révèle de nouveaux usages du droit et de la justice. Cette judiciarisation ne se traduit cependant pas par une meilleure accessibilité des services juridiques qui sont pourtant essentiels pour faire valoir ses droits en justice. Dans cet article, j'avancerai l'idée que cette conjoncture spécifique - judiciarisation de la pauvreté par le droit social ${ }^{1}$ et inaccessibilité des services juridiques — témoigne d'une transformation politique profonde, inscrite dans le néolibéralisme. J'aborderai d'abord l'enjeu du néolibéralisme et ses effets sur le droit et la conception de la justice. Puis, par le biais d'une revue de la littérature, je mettrai en exergue le phénomène de la judiciarisation de la pauvreté par le droit social qui se manifeste, notamment, dans les sphères du logement, de la santé mentale et de protection de la jeunesse. Enfin, j'aborderai l'enjeu de l'inaccessibilité des services juridiques comme entrave au droit à une défense pleine et entière.

\section{L'accumulation par dépossession et la «néolibéralisation » du droit}

Le néolibéralisme est un courant politique caractérisé par la dérégulation des marchés financiers et du travail au détriment des exigences de la vie démocratique. Il est fondé sur l'idée que la dyade libre marché / propriété privée est la meilleure manière d'assurer l'égalité et que les institutions publiques ne peuvent intervenir efficacement en matière économique et sociale. Dans les faits, le néolibéralisme constitue une entreprise d' "accumulation par dépossession": c'est-à-dire l'enrichissement d'une minorité par l'accaparation des avoirs collectifs (Harvey, 2010). La néolibéralisation se matérialise par la réalisation de quatre objectifs transformateurs : la privatisation et l'accaparement des 
biens publics, la financiarisation de l'activité sociale, l'organisation et la gestion de crises économiques et la redistribution stratégique des richesses, notamment aux entreprises privées (Harvey, 2005).

L'accumulation par dépossession explique l'émergence d'une classe d'ultra riches (le fameux "un pour cent») et l'appauvrissement d'une partie de plus en plus importante des populations, notamment la classe moyenne. Depuis une quarantaine d'années, les inégalités ont augmenté dans presque tous les pays, y compris ceux où les politiques sociales sont les plus développées (Word Inequality Lab, 2017). Les inégalités n’en sont pas tant de revenu que de propriété, les plus riches accaparant des proportions de plus en plus importantes du capital privé (Piketty, 2013a). À l'ère néolibérale, il est plus payant d'hériter et de faire fructifier que de travailler (Piketty, 2013b). Ainsi, entre 1980 et 2016, le 1 pour cent a capté 27 pour cent de la croissance pendant que les 50 pour cent les moins riches en captaient 12 pour cent (Word Inequality Lab, 2017).

Au Canada, entre 1980 et 2016, la part du revenu des 10 pour cent les plus aisés dépassait 45 pour cent du revenu national (Word Inequality Lab, 2017). Au Québec, si les inégalités de revenu sont moins importantes que dans le reste du Canada grâce à des mesures fiscales et sociales plus structurantes, elles ne permettent cependant pas de compenser l'augmentation constante des inégalités (Crespo et Rhéault, 2014). Ainsi, entre 1982 et 2010 , le 1 pour cent le plus riche accaparait 11,6 pour cent des revenus avant impôt et 8,3 pour cent des revenus après impôts, en croissance de 60 pour cent sur 17 ans (Institut de recherche et d'information socio-économiques, 2013).

Rappelons qu’au Québec comme ailleurs, les inégalités de revenu se traduisent par des inégalités sociales d'éducation et de santé, et touchent majoritairement des groupes minorisés tels que les femmes, les personnes racisées, LGBTQIA2 ou en situation de handicap (Oxfam, 2018, p. 34; Crenshaw, 1991).

L'accumulation par dépossession et l'accroissement fulgurant des inégalités qui en découle s'opèrent par une mutation des rapports de pouvoir à l'échelle transnationale. Si jusque dans les années 1970, les pays du Nord imposaient leurs volontés au pays du Sud via des politiques nationales postcoloniales, c'est avec les gouvernements néolibéraux de Reagan et de Thatcher qu'un changement s'amorce : les classes dominantes s'allient contre les classes dominées selon un axe nord-sud. Représentation des intérêts des classes aisées devant les institutions financières internationales, initiatives telles que le sommet de Davos, soutien des États du Nord aux classes dirigeantes du Sud, promotion par les agences internationales d'alliances internationales basées sur la rhétorique du libre marché, etc. : le rôle des États est central dans le développement du néolibéralisme (Navarro, 2007). 
Le rôle de l'État néolibéral n'est cependant pas d'assurer des conditions de vie acceptable pour toutes et tous, mais plutôt de maintenir les conditions nécessaires à la bonne marche des activités économiques et la prospérité individuelle. L'organisation et la gestion d'institutions militaires et judiciaires — police, tribunaux, prisons — sont essentielles pour protéger le droit de propriété et soutenir le libre marché (Harvey, 2006). Ainsi, bien que la doctrine néolibérale prône "moins d'État», la diminution de la «taille» des États néolibéraux n'est pas observée sur le terrain, bien au contraire : alors que les budgets alloués à ses missions sociales diminuent, ceux des institutions judiciaires explosent, l’activité de ces institutions visant spécifiquement les groupes sociaux pauvres et précaires (Wacquant, 1998).

Ces changements du rôle étatique ne sont pas sans effet sur le droit et son usage, et il apparaît nécessaire de les envisager comme des mécanismes centraux dans le développement de l'État néolibéral :

Neoliberal claims advance the market side of this contest in capitalist democracies between capitalist imperatives and democratic demands. The contest is persistent because of pressures that capitalist markets make on the legal and political order-pressures not just for familiar protections of property and contract, but also for a favorable return on investment and managerial authority ("freedom to manage"). Neoliberalism, like classical liberalism before it, is also associated with a kind of ideological expansionism, in which market-modeled concepts of efficiency and autonomy shape policy, doctrine, and other discourses of legitimacy outside of traditionally "economic" areas. (Grewal et Purdy, 2014, p. 3)

Outre l'approche répressive de «la loi et l'ordre», la néolibéralisation du droit implique une conception nouvelle de la justice, de l'équité et de l'égalité selon laquelle chacune et chacun est responsable de la revendication et de la mise en ouvre de ses droits en fonction des coûts économiques que représentent les procédures administratives ou judiciaires et des impératifs du marché. Il en découle deux phénomènes. D’une part, une proportion de plus en plus importante de citoyennes et citoyens ne peuvent avoir accès à des services juridiques et font face à la justice sans avocat ou renoncent à leurs droits. D'autre part, des réformes judiciaires d'envergure font la part belle à la liberté contractuelle et la privatisation des processus d'adjudication (Commaille, 2015). 


\section{La justice dans toutes les sphères de la vie. Droit social et judiciarisation de la pauvreté}

Si le droit pénal a été historiquement utilisé pour asservir des groupes sociaux minorisés et marginalisés comme les autochtones (Jaccoud, 1995), les femmes enceintes (Lemonde, 2009) ou les personnes souffrant de troubles mentaux (Laberge et Morin, 1993), des recherches montréalaises récentes semblent pointer dans la même direction. Soulignons parmi d'autres les études de Bellot et Sylvestre (2017) sur l'emprisonnement pour nonpaiement d'amende des personnes en situation d'itinérance, de Sallée et Tschanz (2018) sur le traitement carcéral des jeunes délinquants ou de MacDonald (2016) sur les pratiques de contrôle dans les tribunaux en santé mentale. Ces recherches mettent toutes en lumière comment le statut socio-économique constitue à la fois le point de départ du processus de judiciarisation pénale, mais également le marqueur de l'ensemble de ce processus, depuis la nature des rapports avec les policiers aux expériences correctionnelles. Les pratiques pénales, si elles se sont sophistiquées, semblent donc n'avoir que peu changé : dès le XVIII ${ }^{\mathrm{e}}$ siècle, les pauvres ont été enfermés à la fois pour les punir de leur présumée paresse, mais aussi pour les éloigner de la vue des classes privilégiées pour qui mendicité et précarité sont moralement inadmissibles (Englander, 1998). Cet enfermement s'avère également nécessaire pour favoriser l'activité économique, la présence de pauvres dans un quartier faisant fuir les potentiels clients. Il semble donc que le droit pénal soit depuis longtemps associé aux politiques économiques. Depuis le milieu des années 1970, suivant la théorie $\mathrm{du}$ "carreau brisé » (broken windows), l'enfermement des pauvres se justifie de plus par des arguments sécuritaires associés aux risques et au dérangement (Wilson et Kelling, 1982).

Cependant, d'après Wacquant (2010), l'État néolibéral se développe par un nouvel usage, transversal, des institutions judiciaires et sociales - police, tribunaux, prisons, établissements de santé et de services sociaux, directeur de la protection de la jeunesse (DPJ) ou autorités administratives. Cet usage viserait non seulement la gestion des marginalités ou des dérangements et le risque qu'ils représentent, mais aussi, ou peutêtre d'abord, le maintien des privilèges des classes favorisées par une "pénalisation de la pauvreté» (p. 198) à travers notamment la judiciarisation. Ainsi, les exigences et les normes s'appliquent différemment en fonction du statut socio-économique : "while it embraces laissez-faire at the top, releasing restraints on capital and expanding the life chances of the holders of economic and cultural capital, it is anything but laissez-faire at the bottom" (p. 216). 
Depuis une quinzaine d'années, les recherches québécoises et internationales vont dans le même sens en documentant comment les groupes sociaux qui étaient traditionnellement visés par la judiciarisation pénale subissent maintenant et également une judiciarisation par le droit social. Ces recherches démontrent que des mécanismes judiciaires administratifs et civils pensés pour arbitrer ou protéger sont utilisés à des fins punitives proches des finalités du droit pénal. Si, comme le démontre Bourdieu (2012), la mission sociale de l'État providence — sa "main gauche» — sert des fins de contrôle, ce nouveau phénomène permet de comprendre que nous ne vivons pas un simple retour à l'ère libérale, la logique de marché façonnant dorénavant l'ensemble de la structure étatique, du droit et des politiques sociales (Wacquant, 2010; Brown, 2006).

Je discuterai ici plus précisément des constats de la recherche en matière de logement, de santé mentale et de protection de la jeunesse, puisqu'ils mettent en lumière comment ces mécanismes juridiques constituent implicitement une réponse à la pauvreté. La mise en relation de ces recherches permet aussi de constater comment les interventions judiciaires ciblant certains groupes sociaux ont un effet catalyseur et entrainent la multiplication des procédures judiciaires devant une diversité d'instances. Cette revue de la littérature n'est pas exhaustive et il serait possible de faire les mêmes observations à partir de recherches sur l'immigration, le travail ou la sexualité par exemple (Park, 2011; Barker et Lamble, 2009; Spade, 2015). Cependant, s’intéresser ici au logement, à la santé mentale et à la protection de la jeunesse apparait particulièrement pertinent pour plusieurs raisons. D'abord, la judiciarisation dans ces trois domaines est particulièrement importante : à l'exception des infractions pénales et criminelles, le contentieux à la Régie du logement est le plus important au Québec (Gallié, Brunet et Laniel, 2016a) et le nombre de dossiers judiciaires en santé mentale et en protection de la jeunesse est en progression constante depuis le milieu des années 1990 (Bernheim 2011; Pleau, 2013). Ensuite, le logement étant particulièrement central concernant les conditions de vie, sa mise en péril est susceptible d'avoir des impacts importants sur les autres sphères de la vie personnelle, notamment la santé mentale et les relations familiales (Desmond et Kimbro, 2015). Finalement, le logement étant considéré comme un important facteur d'intégration sociale, l'instabilité locative peut «conduire à la rue, en prison ou à une admission involontaire en psychiatrie» (Dorvil et Morin, 2001 : p. 21), de même qu'à l'intervention du Directeur de la protection de la jeunesse (Bernheim, 2017).

Un retour en arrière est d'abord nécessaire pour bien saisir comment le phénomène de judiciarisation de la pauvreté par le droit social peut aujourd'hui se déployer. Au moment de la création des États-providences, le développement d'un important corpus de droit 
public et administratif doit assurer la prévisibilité des rapports des citoyens avec l'État. La création de services publics va en effet de pair avec la création de cadres normatifs prévoyant leur fonctionnement et le droit privé, jusque-là prédominant, ne peut remplir cette fonction. Dans ce nouvel environnement normatif, les tribunaux occupent une place privilégiée, puisqu'ils sont chargés d'arbitrer les conflits entre les autorités administratives ou publiques et les citoyens. Ces tribunaux sont également considérés comme des lieux neutres d'adjudication permettant à toutes et tous de revendiquer ses droits. C'est ainsi qu'un ensemble de questions sociales - tels le logement, les soins, la protection de la jeunesse, l'aide sociale ou l'assurance automobile — deviennent des questions juridiques, susceptibles d'être judiciarisées.

En matière de logement par exemple, ces États mettent en place des lois contrôlant les hausses des loyers et encadrant le recours à l'éviction, ainsi qu'un système d'assistance sociale locative. L'équilibre entre ces trois composantes du droit du logement devait assurer l'accès à des logements pour toutes et tous dans la mesure où les loyers seraient ainsi abordables et les personnes dans le besoin pourraient toucher des aides. Les propriétaires pourraient éventuellement avoir recours à la procédure judiciaire d'éviction pour nonpaiement de loyer, mais les deux premières mesures étaient censées faire en sorte que la situation ne se présente pas. Or, dans les dernières décennies, le coût de la vie et du logement a fortement augmenté alors que les salaires stagnaient et l'accès aux aides sociales diminuait. Le fragile équilibre qui devait assurer l'accès au logement s'est rompu d'autant plus que la disponibilité des informations sur les antécédents judiciaires permet aux propriétaires de refuser des logements à des personnes ayant déjà été expulsées une première fois, enfermant des familles entières dans la pauvreté (Harlow, 1995; Drapeau, 2007; Desmond 2012, 2016; Desmond et Bell, 2015). Pour Matthew Desmond, qui étudie les évictions locatives aux États-Unis depuis plusieurs années, l'état actuel du droit du logement permet à certains de s'enrichir aux dépens de la précarité des autres. La judiciarisation de la pauvreté, qui passe à la fois par les droits pénal et social, permet ainsi la production et la reproduction des structures sociales inégalitaires (Desmond, 2012 : 121) : «dans les quartiers noirs pauvres, les évictions locatives sont aux femmes ce que l'emprisonnement est aux hommes" (In poor black neighborhoods, what incarceration is to men, eviction is to women, Desmond, 2012, p. 120), un phénomène qui touche particulièrement les familles avec enfants (Desmond et collab., 2013).

Au Québec, les recherches menées par Martin Gallié arrivent à des constats similaires. La Régie du logement reçoit chaque année entre 30000 et 50000 demandes d'expulsion pour non-paiement de loyer. Il va sans dire que «les difficultés financières [des locataires] 
ou les conséquences sociales de leur expulsion [n'étant] pas des questions d'intérêt juridique» (Gallié, Brunet et Laniel, 2016a, p. 641), les juges statuent généralement en faveur des propriétaires. Il existe également une iniquité entre propriétaires et locataires dans le traitement des requêtes : alors que les audiences sur les demandes d'expulsion ont lieu dans les 45 jours en moyenne, lorsque les locataires saisissent la Régie pour insalubrité de leur logement, le délai moyen est de plus de deux ans (Gallié et collab., 2016b). Une étude portant sur la situation de locataires ayant déposé des requêtes à la Régie du logement a démontré que les procédures judiciaires sont anxiogènes et sont directement liées à des états dépressifs, en raison notamment des délais, de la complexité et de l'imprévisibilité des résultats (Breault, 2017).

À partir d'un objet d'étude complètement différent, les recherches menées par Marcelo Otero arrivent aux mêmes conclusions concernant les liens entre précarité locative et santé mentale, et plus particulièrement entre précarité locative et procédures judiciaires concernant la santé mentale. Ses analyses statistiques démontrent qu'à Montréal, la précarité résidentielle est un des principaux marqueurs des interventions de l'urgence psychosociale-justice (UPS-J), plus importante encore que les antécédents psychiatriques ou judiciaires (Otero, 2007). Ces interventions peuvent éventuellement mener à une procédure judiciaire de garde en établissement, soit d'internement psychiatrique. De même, les personnes sans-abris ou évincées de leur logement représentent 20 pour cent des personnes traitées contre leur gré à la suite d'une décision judiciaire (Otero, 2016).

$\mathrm{Au}$ Québec, la judiciarisation des procédures d'internement psychiatrique et de soins imposés au début des années 1990 devait permettre un changement de culture dans les milieux d'intervention et la protection des droits des patientes et patients. Il n'était dorénavant plus question de prendre des décisions dans leur intérêt clinique, mais bien sur la base des droits, à la suite d'un procès civil en bonne et due forme. Les deux procédures sont entièrement étanches : la garde en établissement ne peut avoir lieu qu'en cas de danger pour soi ou autrui et les soins ne peuvent être imposés que dans la mesure où la personne concernée est inapte à y consentir et qu'ils sont requis par l'état de santé. Dans tous les cas, la décision judiciaire ne peut être prise qu'après avoir pris connaissance de l'ensemble de la preuve et entendu la partie défenderesse, qui peut être représentée par avocate ou avocat et avoir des témoins.

Les recherches démontrent non seulement la surreprésentation des personnes vivant dans la pauvreté parmi les défenderesses ou défendeurs, mais également que le style de vie - état du logement, situation scolaire et d'emploi, état des relations avec les proches ou consommation - est un élément déterminant des décisions d'ordonnances 
de garde en établissement et de soins (Bernheim, 2011). Les audiences sont l'occasion de discuter des difficultés quotidiennes des défenderesses ou défendeurs, qu'elles soient liées à l'alimentation, au logement ou à l'utilisation de l'espace public, et qui font bien souvent partie des éléments expliquant leur présence à l'hôpital, et particulièrement en psychiatrie. Ainsi, des altercations dans les maisons de chambre, les habitations à loyers modiques (HLM) ou les espaces publics, le fait de consommer à l'extérieur, de vivre dans un appartement insalubre, dans un squat ou à la rue peut déboucher sur des procédures judiciaires de garde en établissement ou de soins. Il s'agit donc pour les tribunaux de gérer le risque et le dérangement associés à la précarité.

Bien que les défenderesses et défendeurs expliquent aux juges les difficultés liées à l'hospitalisation de longue durée - telles que la perte de leur logement, de leur emploi et de leurs relations sociales, le vol de l'ensemble de leurs biens dans leurs logements ou l'impossibilité de rester impliqués dans certaines activités - les tribunaux semblent considérer que, vu l'état de pauvreté dans lequel elles ou ils vivent, le préjudice n'est pas tellement important; au contraire. Certains juges affirment même qu' «ici on ne rend pas justice, on rend service» et que «la décision est prise avec le cour» (Bernheim, 2011, p. 535; p, 671). La justice semble donc avoir pour certaines et certains des vertus thérapeutiques et proposer des solutions viables à des personnes en détresse en raison de leurs conditions de vie.

Cette conception de la justice en tant qu'instance de règlement des problèmes sociaux trouve un certain écho dans la littérature. Deux pistes sont développées à cet égard. La première est celle de la reprise de pouvoir par le droit (empowerment), selon laquelle les droits peuvent constituer des outils de transformation sociale, et notamment d'éradication de la pauvreté (Banik, 2008). Il suffirait donc d'éduquer au droit, l'appropriation du discours sur les droits constituant en soi une forme de reprise de pouvoir sur sa propre vie. La seconde piste est celle de la jurisprudence thérapeutique, selon laquelle le droit, les juristes et les tribunaux sont des agents thérapeutiques qui peuvent agir sur la vie sociale notamment via les institutions judiciaires (Wexler, 1994). Elle soutient des initiatives de transformation de la justice comme le développement actuellement en vogue de tribunaux pénaux spécialisés, entre autres, en santé mentale, en itinérance ou en toxicomanie. Ces deux doctrines ont en commun de ne pas remettre en question la dimension coercitive et l'effet préjudiciable du processus judiciaire.

Les personnes faisant l'objet de demandes de garde en établissement et de soins, sont pourtant souvent judiciarisées devant d'autres tribunaux pour des faits souvent similaires, que ce soit la Cour municipale, la Chambre criminelle et pénale de la Cour du Québec, 
la Régie du logement, le Tribunal administratif du Québec ou la Chambre de la jeunesse de la Cour du Québec. Elles ont reçu des contraventions, sont en non-respect des conditions, font l'objet de demandes d'expulsion de leur logement, sont poursuivies par l'aide sociale ou ont un dossier ouvert par le DPJ (Bernheim, 2011). Cette dernière situation touche principalement les femmes pour qui le maintien de la garde des enfants est difficile après un internement psychiatrique comme le rapportait une étude du ministère de la Santé et des Services sociaux en 1997.

Ce constat n'est pas surprenant. Environ 45 pour cent des familles auprès desquelles le DPJ intervient vit de l'aide sociale, plus de 50 pour cent déclare un revenu de moins de 15000 dollars et la moitié est monoparentale (Protecteur du citoyen, 2013). Pour certains auteurs, les interventions du DPJ visent à «compenser les carences éducatives attribuées aux parents, en particulier qui appartiennent à des milieux socio-économiquement faibles" (Boulanger, Larose et Couturier, 2010, p. 154). À cet égard, l'étude de la jurisprudence de la Chambre de la jeunesse démontre que l'instabilité résidentielle est un enjeu récurrent dans les décisions de placement. L'instabilité résidentielle, les dettes, l'absence de travail et le style de vie sont ainsi régulièrement discutés sans mise en contexte économique. Dans plusieurs cas, le tribunal attribue la situation à des choix personnels allant jusqu'à affirmer par exemple que "la mère n'a pas changé son mode de vie : elle est encore instable sur le plan du logement, ayant déménagé au moins neuf fois depuis le prononcé de la dernière ordonnance» (X (Dans la situation de), 2006, parag. 22). Les décisions relatent des conditions de vie très précaires : appartements peu meublés "malgré les recommandations des intervenants sociaux" (Protection de la jeunesse - 072127, 2007, parag. 22), alimentation "déficiente» ou peu variée (Protection de la jeunesse 09430, 2009, parag. 14). L'incapacité d'offrir un confort matériel considéré minimal par les tribunaux - mise à la disposition des enfants d'espaces personnels de travail et de jeu, repas équilibrés - compromet la possibilité pour les parents de conserver la garde de leurs enfants (Bernheim, 2017).

Bien que les signalements n'aient que peu augmenté, soulignons que le volume des dossiers judiciaires a crû d'environ 20 pour cent entre 1994 et 2007 (Pleau, 2013; Ministère de la Justice, 2004). La Loi sur la protection de la jeunesse prévoit pourtant explicitement que le recours judiciaire doit être exceptionnel, mais cette augmentation semble correspondre à celle qui est constatée dans d'autres domaines du droit social, notamment en santé mentale (Commaille, 2015; Bernheim et Commaille, 2012; Bernheim, 2011). Elle témoigne également d'un changement dans les objectifs de l'intervention : il ne s'agirait plus de tenter de répondre aux besoins des familles en 
difficulté, mais plutôt de gérer le risque qu'elles représentent — risques de transmission intergénérationnelle de la pauvreté, mais aussi de la délinquance (Featherstone, White et Morris, 2014a; Cardi 2007). Les familles vivant dans la pauvreté sont envisagées comme responsables de leur propre situation et doivent impérativement se réformer pour garder le contact avec leurs enfants, notamment en assurant les infrastructures matérielles nécessaires à leur bien-être. Les ordonnances judiciaires les contraignent à se soumettre à un ensemble d'exigences comme consulter des professionnels de la santé, subir des évaluations et prendre des médicaments, subir des tests de dépistage aléatoires ou recevoir des visites surprises, en dépit de leurs droits à consentir aux soins, à la vie privée et à la liberté (Bernheim 2017; Featherstone, White et Morris, 2014b).

Expulsions locatives, internements, soins imposés et placement d'enfants : il apparaît qu'une première procédure judiciaire est bien souvent le début d'une longue série. Devant les tribunaux, être pauvre est à la fois une offense et une responsabilité dont il faut s'expliquer, une situation d'autant plus kafkaïenne que les services juridiques qui permettraient la mise en œuvre du droit à une défense pleine et entière sont difficilement accessibles.

\section{Se battre à armes inégales dans toutes les sphères de sa vie. Judiciarisation de la pauvreté et inaccessibilité des services juridiques au Québec}

Au Québec, le système de justice est contradictoire. Il est donc fondé sur les droits des deux parties à une procédure de participer au débat judiciaire et sur leur capacité égale de présenter de la preuve et des arguments juridiques. Â part de rares exceptions, les avocates et avocats ${ }^{2}$ sont les seuls à pouvoir donner des conseils juridiques, rédiger des procédures et faire de la représentation devant les tribunaux. Leur rôle est donc central tant à l'égard des parties que de la magistrature : d'une part elles ou ils assistent et représentent les intérêts de leurs clientes et clients et d'autre part, elles ou ils préparent les dossiers et les débats judiciaires afin de permettre aux tribunaux de trancher sans s'immiscer dans les procédures.

Dans les dernières décennies, l'accès aux services juridiques s'est considérablement réduit. Pour plusieurs, ce phénomène est directement lié à l'augmentation des honoraires d'avocate ou avocat qui se situaient en moyenne entre 151 \$ et 200 \$ de l'heure en 2015 (Barreau du Québec, 2015). Comparativement, le revenu moyen des Québécoises et 
Québécois était de 26857 \$ par année en 2016 (Institut de la statistique du Québec, 2016).

Une des solutions à cette inaccessibilité est le programme d'aide juridique, soit l'accès à une avocate ou à un avocat rémunéré en tout ou en partie par l'État. À l'image de l'ensemble des services publics, l'admissibilité à l'aide juridique est déterminée par des critères de plus en plus restrictifs. Les seuils de revenu fixant l'admissibilité n'ont pas été indexés entre 1982 et 2012 et sont actuellement en rattrapage. Depuis mai 2018, l'accès aux services juridiques gratuits est réservé aux personnes gagnant $21840 \$$ et moins pour une personne seule, et 28525 \$ et moins pour un adulte seul avec deux enfants ou plus (Règlement sur l'aide juridique, art 18). Au-delà de ces revenus, des services peuvent être offerts moyennant le paiement d'une franchise. Le gouvernement québécois considère pourtant qu'une personne seule gagnant jusqu'à $24400 \$$ et une famille de trois personnes vivant avec moins de 37345 \$ est une famille à faible revenu (Gouvernement du Québec, 2018).

Soulignons par ailleurs que l'aide juridique est offerte soit par des avocates ou avocats salariés de l'État soit par des avocates et avocats prenant des mandats privés d'aide juridique. Les premières ou premiers cumulent un nombre important de dossiers : en 2017, 100279 dossiers ont été traités par les 385 avocates et avocats permanents de l'aide juridique (Commission des services juridiques, 2018). Les secondes ou seconds travaillent à de bas tarifs créant une instabilité financière et obligeant le travail bénévole pour remplir les mandats. De moins en moins d'avocates et d'avocats acceptent des mandats privés d'aide juridique : en 25 ans, leur proportion a baissé du tiers. Ainsi, le rattrapage des seuils d'admissibilité «ne permet pas d'améliorer à [lui seul] l'accessibilité à la justice» (Jeune Barreau de Montréal, 2016, p. 3).

Le programme d'aide juridique ne couvre pas tous les domaines de droit et toutes les situations. La couverture est complète en cas de risque de perte de liberté (emprisonnement ou garde en établissement) et de mise en péril de la sécurité physique, psychologique ou des moyens de subsistance. Les procédures concernant les régimes de protection (curatelles, tutelles), les soins, la protection de la jeunesse sont couvertes, de même que certaines procédures criminelles, administratives (aide sociale, rentes, etc.), en matière familiale et en vertu de certaines lois spécifiques. Les recours de moins de 15000 à la Régie du logement sont exclus. Le régime d'aide juridique peut permettre de protéger les droits d'une personne accusée ou poursuivie; il ne permet cependant pas d'en revendiquer. Une recherche récente démontre que les avocates et avocats considèrent généralement la couverture de l'aide juridique insuffisante et qu'elles et ils contestent 
le caractère discrétionnaire de la détermination des matières couvertes. De même, si le programme d'aide juridique permet de faire appel aux services d'experts judiciaires, les tarifs assumés par l'État ne reflètent pas ceux du marché (Jeune Barreau de Montréal, 2016). Le recours à l'expertise est pourtant essentiel dans certains types de dossiers et son absence compromet la possibilité de faire valoir ses droits. Dans le cas des demandes de garde en établissement, de soins ou de protection de la jeunesse, si l'expertise n'est pas essentielle, les établissements de santé et le DPJ en disposent systématiquement, ce qui crée un évident déséquilibre des forces.

Étant donné les limites des critères d'admissibilité et de couverture du programme d'aide juridique et les frais associés aux services juridiques privés, un nombre de plus en plus important de personnes se présentent en justice sans avocat. 55 pour cent des dossiers en matière civile concernent au moins une partie non représentée (Ministère de la Justice du Québec, 2016), un chiffre pouvant aller jusqu’à 40 pour cent dans les dossiers en matière criminelle (Ministère de la Justice du Canada, 2002). Ces personnes n'ont d'autre choix que de se tourner vers des services communautaires ou encore de chercher de l'information juridique de manière autonome, en ligne par exemple. Dans tous les cas, elles n'ont généralement pas accès à des conseils juridiques.

Au Québec en effet, seuls les membres des ordres professionnels de juristes (Barreau et Chambre des notaires) peuvent donner des conseils juridiques - soit une opinion concernant une situation spécifique. Les étudiantes et étudiants en droit, les parajuristes et les intervenantes et intervenants des organismes communautaires, au même titre que n'importe qui, ne peuvent fournir que de l'information juridique, soit le simple contenu des dispositions législatives et réglementaires.

Dans les dernières années, pour faire face à la multiplication des parties non représentées devant les tribunaux, les services d'information juridique se sont largement développés, que ce soit à l'initiative du ministère de la Justice ou d'organisations privées ou communautaires : soulignons notamment la création des Centres de justice de proximité, la mise à disposition de dépliants informatifs dans les Palais de justice ou sur les sites internet des tribunaux, les services de préparation à l'audience offerts par les Barreaux ou les Jeunes Barreaux ou encore les services offerts par des organismes comme les cliniques juridiques des différentes universités. L'accès à ces services d'information requiert le plus souvent des compétences en lecture, alors que 53 pour cent de la population québécoise est considérée comme analphabète fonctionnelle (Institut de la statistique du Québec, 2017).

Les retombées positives de ces services d'information pour les personnes n'ayant pas accès à des services juridiques, notamment en cour, ne sont pas clairement établies. 
Depuis plusieurs années, les recherches démontrent plutôt que la simple information ne permet pas de remplacer le travail des professionnelles et professionnels du droit et que les personnes non représentées n'ont généralement pas gain de cause devant les tribunaux, peu importe qu'elles aient eu accès ou non à de l'information juridique (Hugues, 2013; Birnbaum, Bala et Bertrand, 2012; Vayda et Ginsberg, 2012). Des clientes et des clients d'une clinique juridique de Montréal ont par exemple rapporté que l'information juridique est source de confusion, ne sachant pas comment l'appliquer à leur propre situation ni comment la présenter à la cour. La dimension technique du droit peut faire en sorte que, malgré la préparation, des erreurs de vocabulaire, de moment ou d'ordre de présentation des arguments juridiques peuvent faire toute la différence (Équipe de recherche du Chantier autoreprésentation et plaideur citoyen, 2018). Il a en effet été démontré que le niveau de maîtrise du langage, du raisonnement et du formalisme juridiques a des répercussions sur le cours des audiences judiciaires (Robertson et Giddings, 2014; Bernheim, Laniel et Jannard, 2018).

La rigidité du cadre juridique québécois constitue une contrainte réelle pour les organismes qui souhaiteraient développer des services au-delà de la simple information, à moins de disposer de l'expertise de juristes membres du Barreau ou de la Chambre des notaires. Outre le risque de poursuites pour pratique illégale du droit, la difficulté réside dans le flou entre information et conseil juridiques. S'il est admis que l'information juridique porte sur le droit en général alors que le conseil juridique porte sur une situation particulière, cette distinction doit néanmoins être évaluée au cas par cas (Charlebois c Barreau du Québec, 2012). Il est évident que pour des organismes peu financés, disposant de peu de ressources et faisant face aux besoins spécifiques de leur clientèle, il est difficile, voire impossible, dans le feu de l'action des consultations ou des accompagnements, de ne pas glisser dans le conseil juridique. Pour les organismes bénéficiant des services d'avocates et d'avocats, cette situation les contraint à intervenir auprès de chaque cliente et client à chaque étape des consultations ou des suivis de dossier, plutôt que de se consacrer à des tâches plus complexes (Équipe de recherche du Chantier autoreprésentation et plaideur citoyen, 2018).

Par comparaison, dans plusieurs provinces canadiennes, sans être membres du Barreau, les étudiantes et étudiants en droit peuvent poser certains actes juridiques sous la supervision d'avocates ou avocats en pratique, tels que donner des conseils, assister dans la rédaction de formulaires et préparer à des audiences. De même, pour certaines procédures, les parajuristes peuvent donner des conseils et représenter des clientes et clients en cour. Plus souples, ces cadres juridiques favorisent le développement d'initiatives mobilisant des 
étudiantes, étudiants ou parajuristes, telles que les ateliers de rédaction de requêtes offerts à Vancouver par des parajuristes, l'assistance à la rédaction de formulaires de demande d'asile par les étudiantes et étudiants de l'Université de Moncton ou la représentation devant certains tribunaux administratifs par des étudiantes et étudiants de la Walk-In Advocacy Clinic à Saskatoon. Plusieurs services visent des clientèles particulièrement marginalisées, entre autres, les personnes trans, les femmes victimes de violences conjugales ou les personnes faisant usage de drogues (Laniel, Bahary-Dionne et Bernheim, 2018; Équipe de recherche du Chantier autoreprésentation et plaideur citoyen, 2019).

De même, devant plusieurs tribunaux canadiens, diverses initiatives sont prises pour assister et soutenir les personnes non représentées dans leur parcours judiciaire telles que les "amis McKenzie» (McKenzie Friends) qui proposent des accompagnements à la cour. Non nécessairement des juristes, ces derniers peuvent notamment aider les personnes non représentées à organiser leurs documents légaux, s'asseoir à côté d'elles en salle d'audience, prendre des notes pour elles et leur offrir un soutien moral. Cette pratique n'est cependant généralement pas reconnue par les tribunaux québécois (Laniel, Bahary-Dionne et Bernheim, 2018; Équipe de recherche du Chantier autoreprésentation et plaideur citoyen, 2019).

Dans un contexte de judiciarisation de la pauvreté, au Québec plus quailleurs, l'inaccessibilité des services juridiques conjuguée à la rigidité du cadre légal en matière de compétences réservées a pour effet de compromettre le droit à une défense pleine et entière, d'autant plus que l'État et les institutions publiques ou parapubliques - municipalité, directeur des poursuites criminelles et pénales (DPCP), autorités administratives, établissements de santé et de services sociaux, DPJ — sont automatiquement représentés par procureurs et disposent des services d'experts judiciaires. Dans ces cas, alors que David rencontre Goliath, l'expérience judiciaire s'avère des plus éprouvantes (Bernheim, Laniel et Jannard, 2018).

\section{Conclusion. Néolibéralisme et justice productrice d'inégalités}

Les droits sont généralement envisagés comme permettant d'accéder à plus d'égalité. Après la Seconde Guerre mondiale, et pour quelques décennies, le développement d'instruments internationaux et nationaux de reconnaissance des droits s'est concrétisé en changements politiques et sociaux importants. Au Québec, les exemples sont nombreux, telle la reconnaissance de la capacité juridique aux femmes mariées en 1964, de l'égalité entre les époux en 1969, de droits aux enfants illégitimes en 1970 ou de l'obligation 
d'obtenir le consentement avant tout acte médical en 1971. Plus près de nous, des luttes importantes ont été menées, et gagnées grâce aux Chartes, entre autres, celles menant au droit à l'avortement et au suicide assisté ou à l'ouverture de sites d'injection supervisée.

Force est de constater que les droits n'ont été que de peu d'utilité en matière de conditions de vie. La Loi visant à lutter contre la pauvreté et l'exclusion sociale adoptée en 2002, fruit d'une longue revendication, n'a pas empêché les coupures sauvages dans les programmes sociaux ni la mise en place de conditions d'admissibilité à l'aide sociale de plus en plus restrictives. Elle n'est d'aucun secours par rapport à la précarisation des emplois, la hausse continuelle du coût de la vie, la gentrification ou l'absence de garderies et de cliniques dans les quartiers pauvres. Les recours devant les tribunaux, y compris sur la base des Chartes, n'ont jusqu'à maintenant donné aucun résultat.

Non seulement les droits et la justice n'ont pas pu permettre d'assurer des conditions de vie acceptables pour toutes et tous, mais les constats de la recherche actuelle sur la judiciarisation de la pauvreté permettent de démontrer que les tribunaux jouent aujourd'hui un rôle de reproduction des inégalités, au-delà du droit pénal. Ainsi, il n’apparait plus utile de séparer les différents domaines de droit pour en comprendre la dynamique; il convient au contraire de comprendre le système judiciaire comme un ensemble produisant des effets préjudiciables de nature à infléchir le cours d'une trajectoire de vie.

Le droit social censé assurer les missions sociales de l'État sert aujourd'hui à des recours fondés sur la base du risque dont la dimension pénalisante est importante (entre autres, internement, soins imposés, suivis par le DPJ, mais également coupures d'allocation ou refus de demande d'asile). En outre, ce sont les conditions dans lesquelles cette judiciarisation s'exerce qui produisent des effets structuraux inégalitaires. Les conditions restrictives d'admissibilité au programme d'aide juridique et les limites de la couverture ainsi que l'absence de services juridiques gratuits ou abordables impose pour plusieurs la responsabilité irréaliste de maitriser le langage, le formalisme et la technicité du droit pour espérer faire valoir ses droits. Pour les autres, il faut composer avec des avocates ou avocats débordés, souvent sous-payés et disposant de peu de ressources. La judiciarisation de la pauvreté n'est définitivement pas un combat à armes égales.

La néolibéralisation du droit et la judiciarisation sont des dimensions incontournables de l'intervention en matière de pauvreté et la recherche en la matière doit impérativement s'y intéresser. Dans un premier temps, il semble que réfléchir le droit et le recours aux tribunaux par domaine ou par matière juridique (droit pénal/civil/administratif) ne permette pas de capter la complexité des trajectoires des personnes à travers le système 
de justice. Il apparaît dans le contexte actuel beaucoup plus utile de se concentrer sur les trajectoires des personnes judiciarisées pour considérer la nature, la fréquence et les effets des contacts avec l'institution judiciaire. Dans un second temps, il importe de changer de regard sur le processus judiciaire pour en considérer les effets coercitifs et structuraux. Alors que l'usage du droit social et des processus judiciaires qui y sont associés a changé dans les dernières années, il apparaît pertinent de remettre en question l'idée que le recours aux tribunaux correspond à une protection ou à une mise en œuvre des droits pour considérer le droit et la judiciarisation comme producteurs d'inégalités. Il est évident en effet que lorsque les personnes en cause ne sont pas en mesure de se défendre adéquatement, le droit et la justice ne contribuent qu’à renforcer les inégalités existantes. Finalement, il faut considérer la dimension méthodologique de ces pistes de recherche qui nécessitent une grande proximité avec les personnes judiciarisées. L'étude des trajectoires individuelles dans le système de justice ne peut s'envisager que par des approches qualitatives inductives comme l'ethnographie, y compris l'accompagnement dans les démarches administratives et judiciaires, et les parcours de vie (Fortin, 2018; Bernheim, 2015).

Dans la mesure où la recherche, et plus particulièrement la recherche en sciences sociales, doit contribuer à l'émancipation en rendant visible et en mettant en cause les rapports de pouvoir (Acker, Barry et Esseveld, 1991), cet article a deux objectifs. Le premier est d'amorcer une analyse établissant des liens entre différentes situations de judiciarisation de la pauvreté qui sont, à toute évidence, liées. À défaut d'une analyse systématique de trajectoires, il apparaît pertinent de considérer les résultats de recherche de manière dynamique, en envisageant les contacts judiciaires comme autant de ruptures interreliées de trajectoire (Bessin, Bidart et Gossetti, 2010). Le second est de mettre ces résultats de recherche dans leur contexte, qui est celui de l'inaccessibilité des services juridiques. En effet, bien que les questions d'accès à la justice soient très à la mode, il n'existe actuellement que peu de réflexion structurelle sur le sujet. Or une telle réflexion est aujourd'hui plus que nécessaire et urgente.

\section{Bibliographie}

ACKER, Joan, Kate BARRY et Johanne ESSEVELD (1991). «Objectivity and truth: Problems in doing feminist research ", dans Mary Margaret FONOW, et Judith COOK (dires.), Beyond methodology: Feminist scholarship as lived research, Indiana University Press, p. 133-153.

BANIK, Dan (2008). Rights and Legal Empowerment in Eradicating Poverty, Surrey, Ashgate, 268 p. 
BARKER, Nicholas, et Sarah LAMBLE (2009). «From social security to individual responsibility: sanctions, conditionality and punitiveness in the Welfare Reform Bill 2009 (Part One) ", Journal of Social Welfare and Family Law, Vol 31, N³, p. 321-332.

BARREAU DU QUÉBEC (2015). Barreau-mètre 2015 : la profession en chiffres, réf. du 15 octobre 2018, https://www.barreau.qc.ca/pdf/publications/barreau-metre-2015.pdf

BECKER. Howard (1963). Outsiders, studies in the sociology of deviance, New York, Free Press of Glencoe, 179p.

BELLOT, Céline, et Marie-Ève SYLVESTRE (2017). «La judiciarisation de l'itinérance à Montréal : les dérives sécuritaires de la gestion pénale de la pauvreté», Revue générale de droit, Vol. 47, p. 11-44.

BERNHEIM, Emmanuelle, Richard-Alexandre LANIEL et Louis-Philippe JANNARD (2018). «Les justiciables non-représentés face à la justice : une étude ethnographique du Tribunal administratif du Québec», Windsor review of legal and social issues, Vol. 39, p. 67-104.

BERNHEIM, Emmanuelle (2017). «Sur la réforme des mères déviantes : les représentations de la maternité dans la jurisprudence de la Chambre de la jeunesse, entre différentiation et responsabilisation", Revue générale de droit, Vol. 47, p. 45-75.

BERNHEIM, Emmanuelle (2015). «De petite fille abusée à mère négligente : protection de la jeunesse et matrice de domination", Revue canadienne femmes et droit, Vol. 27, N², p. 184-206.

BERNHEIM, Emmanuelle (2011). Les décisions d'hospitalisation et de soins psychiatriques sans le consentement des patients dans des contextes clinique et judiciaire - Une étude du pluralisme normatif appliqué, [thèse de doctorat], Montréal, Canada, Université de Montréal et Cachan, France, École normale supérieure, $852 \mathrm{p}$.

BERNHEIM, Emmanuelle, et Jacques COMMAILLE (2012). «Quand la justice fait système avec la remise en question de l'état social», Droit et société, Vol. 81, N² 2, p. 281-298.

BESSIN, Marc, Claire BIDART et Michel GROSSETTI (dirs) (2010). Bifurcations: les sciences sociales face aux ruptures et à l'évènement, Paris, La Découverte, 402 p.

BIRNBAUM, Rachel, Nicholas BALA et Lorne BERTRAND (2012) «The rise of self representation in Canada's Family Court: The complex picture revealed in surveys of jugdes, lawyers and litigants ", Revue du Barreau Canadien, Vol. 91, N 1, p. 67-95.

BLACK, Donald (1983). "Crime as Social Control», American Sociological Review, Vol. 48, N¹, p. 34-45.

BOULANGER, Dany, François LAROSE et Yves COUTURIER (2010). «La logique déficitaire en intervention sociale auprès des parents : les pratiques professionnelles et les représentations sociales ", Nouvelles pratiques sociales, Vol. 23, N 1, p. 152-176.

BOURDIEU, Pierre (2012). Sur l'État - Cours au Collège de France 1989-1992, Paris, Seuil, 656 p. 
BREAULT, Geneviève (2017). Étude des conséquences psychosociales des conflits en habitation chez les personnes locataires demanderesses de services-conseils d'une ressource communautaire montréalaise, [thèse de doctorat], Montréal, Canada, Université du Québec à Montréal, 274 p.

BROWN, WENDY (2006). "American nightmare: Neoliberalism, neoconservatism, and dedemocratization", Political Theory, Vol. 34, N 6, p. 690-714.

CARDI, Coline (2007). «La "mauvaise mère" : figure féminine du danger ", Mouvements, Vol. 49, $\mathrm{N}^{\circ}$ 1, p. 27-37.

CHARLEBOIS C. Barreau du Québec, 2012 QCCA 788

COMMAILLE, Jacques (2015). À quoi nous sert le droit?, Paris, Gallimard, 528 p.

COMMISSION DES SERVICES JURIDIQUES (2018). $46^{\circ}$ rapport annuel de gestion, Montréal, réf. du 12 mai 2019, https://www.csj.qc.ca/IntranetUploads/CSJ/Francais/Fichiers/final_Rapport_ Annuel_18-06_LR.PDF

CRENSHAW, Kimberley (1991). "Mapping the margins: Intersectionality, identity politics, and violence against women of color", Stanford Law Review, Vol. 43, № 6, p. 1241-1299

CRESPO, Stéphane, et Sylvie RHÉAULT, (2014). L’inégalité du revenu disponible des ménages au Québec et dans le reste du Canada : bilan de 35 années, [rapport de recherche], Québec, Institut de la statistique du Québec, 15 p.

DESMOND, Matthew, et Rachel Tolbert KIMBRO (2015). «Eviction's fallout: Housing, hardship, and health", Social Forces, Vol. 94, $\mathrm{N}^{\circ} 1$, p. 2-11.

DESMOND, Matthew, et Monica BELL (2015). «Housing, poverty, and the law» (2015) Annual Review of Law and Society Science, Vol 11, p. 15-35.

DESMOND, Matthew, et collab. (2013). «Evicting children», Social Forces, Vol. 92, №1, p. 303-327.

DESMOND, Matthew (2012). «Eviction and the reproduction of urban poverty», American Journal of Sociology, Vol. 118, No 1, p. 88-133

DORVIL, Henri, et Paul MORIN (2001). «Présentation : Multiples enjeux et perspectives diverses», Nouvelles pratiques sociales, Vol. 14, №2, p. 20-27.

DRAPEAU, Marie-Hélène (2007). Lutte pour le droit au logement et perspective de déconstruction du pouvoir dans un espace autogéré: le squat du 920 de la Chevrotière, [mémoire de maitrise], Québec, Canada, Université Laval, 224 p.

ENGLANDER, David (1998). Poverty and poor law reform in nineteenth-century Britain, 1834-1914, Londres, Routledge, $152 \mathrm{p}$.

ÉQUIPE DE RECHERCHE DU CHANTIER AUTOREPRÉSENTATION ET PLAIDEUR CITOYEN (2019). Parajuristes, étudiant.es en droit et ami.es McKenzie : quel rôle en matière d'accès à la justice?, [rapport de recherche], Montréal, $46 \mathrm{p}$. 
ÉQUIPE DE RECHERCHE DU CHANTIER AUTOREPRÉSENTATION ET PLAIDEUR CITOYEN (2018). La force du suivi personnalisé pour les personnes autoreprésentées, [rapport de recherche], Montréal, $30 \mathrm{p}$.

FEATHERSTONE, Brid, Kate WHITE et Sue MORRIS (2014a). Re-imaginig child protection Towards humane social work with families, Chicago, University of Chicago Press, $256 \mathrm{p}$.

FEATHERSTONE, Brid, Kate WHITE et Sue MORRIS (2014b). «A marriage made in hell: early intervention meets child protection", British Journal of Social Work, Vol. 44, N 7, p. 1735-1749.

FORTIN, Véronique (2018). «The Control of public spaces in Montreal in times of managerial justice», Champ pénal, Vol. 25, réf du 25 février 2019, https://journals.openedition.org/champpenal/10115.

FOSCARINIS, Maria (1996). "Downward spiral: Homelessness and its criminalization», Yale Law and Policy Review, Vol. 14, $\mathrm{N}^{\circ} 1$, p. 1-63.

FOUCAULT, Michel (1975). Surveiller et punir, Paris, Gallimard, 352 p.

GALANTER, Marc (1983). «Making law for the oppressed», The Other Side, Vol. 3, N², p. 7-15.

GALLIÉ, Martin, Julie BRUNET et Richard-Alexandre LANIEL (2016a). "Les expulsions pour arriérés de loyer au Québec : un contentieux de masse", Revue de droit de McGill, Vol. 61, N 3, p. 611-666.

GALLIÉ, Martin, et collab. (2016b). «Les délais judiciaires comme modalité d'organisation d'une justice de classe : la hiérarchisation des causes à la Régie du logement» (2016) Nouveaux Cabiers du socialisme, Vol. 16, p. 91-100.

GOUVERNEMENT DU QUÉBEC (2018). Seuils de faible revenu (SFR) en vigueur pour la période estivale, réf. du 18 octobre 2018, http://www.vacancesfamiliales.qc.ca/seuils-de-faible-revenu-2018.pdf

GREWAL, David Singh, et Jedediah PURDY (2014). «Introduction: Law and neoliberalism ", Law and Contemporary Problems, Vol. 77, $\mathrm{N}^{\circ} 1$, p. 1-23.

HARLOE, Michael (1995). The People's home?: Social rented housing in Europe and America, Oxford, Blackwell Publisher, 586 p.

HARVEY, David (2010). «The Right to the city: From capital surplus to accumulation by dispossession ", dans Swapna BANERJEE-GUHA (dire.), Accumulation by Dispossession: Transformative Cities in the New Global Order, New Dehli, Sage Publication, p. 17-32.

HARVEY, David (2006). "Neo-liberalism as creative destruction», Geografiska Annaler. Series B, Human Geography, Vol. 88, № 2, p. 145-158

HARVEY, David (2005). A Brief history of neoliberalism, Oxford, Oxford University Press, 256 p. 
INSTITUT DE LA STATISQUE DU QUÉBEC (2017). Rapport québécois du Programme pour l'évaluation internationale des compétences des adultes (OCDE), Québec, réf. du 25 février 2019, http://www.stat.gouv.qc.ca/statistiques/education/alphabetisation-litteratie/peica.pdf

INSTITUT DE LA STATISQUE DU QUÉBEC (2016). "Revenu disponible», Bulletin Flash, réf. du 25 septembre 2018,

http://www.stat.gouv.qc.ca/statistiques/economie/comptes-economiques/revenu-menage/revenudisponible-2016.pdf

INSTITUT DE RECHERCHE ET D'INFORMATION SOCIO-ÉCONOMIQUE (2013). Note socio-économique - Les inégalités : le 1\% au Québec, réf. du 5 octobre 2018, https://cdn.irisrecherche.qc.ca/uploads/publication/file/Note-1 pourcent-WEB.pdf

HUGUES, Patricia (2013). "Advancing access to justice through generic solutions: The risk of perpetuating exclusion", Recueil annuel de Windsor d'accès à la Justice, Vol. 31, N 1, p. 1-22.

JACCOUD, Mylène (1995). «L/Histoire de limposition du processus pénal au Nunavik (NouveauQuébec inuit)», The Journal of Human Justice, Vol. 6, N² 2, p. 105-130.

JEUNE BARREAU DE MONTRÉAL (2016). Rapport du JBM sur le système d'aide juridique québécois, Montréal, $64 \mathrm{p}$.

LABERGE, Danielle, et Marie ROBERT (1992). «La judiciarisation de la santé mentale : profil des personnes accusées devant la cour municipale de Montréal», Nouvelles pratiques sociales, Vol. 5, $\mathrm{N}^{\circ} 1$. p. 31-46.

LABERGE, Danielle, et Daphné MORIN (1993). "Troubles mentaux et intervention pénale. Questions entourant les évaluations de la judiciarisation en Amérique du Nord ", Déviance et société, Vol. 17, $\mathrm{N}^{\circ} 3$, p. 309-348.

LANIEL, Richard-Alexandre, Alexandra BAHARY-DIONNE et Emmanuelle BERNHEIM (2018). "Agir seul en justice : du droit au choix - État de la jurisprudence sur les droits des justiciables non représentés", Les Cahiers de droit, Vol. 59, N³, p. 495-532.

LEMONDE, Lucie (2009). "Les menaces au droit à l'avortement et à l'autonomie des femmes enceintes", Les Cahiers de Droit, Vol. 50, N³-4, p. 611-635.

NAVARRO, Vicente (2007). «Neoliberalism as a class ideology: Or the political causes of the growth of inequalities", International Journal of Health Services, Vol. 37, N 1, p. 47-62.

MACDONALD, Sue-Ann (2016). «Regulating madness in a mental health court», TSANTSA, Vol. 21, p. 26-37.

MINISTÈRE DE LA JUSTICE DU Canada (2002). Étude nationale sur les adultes non représentés accusés devant les cours criminelles provinciales - Partie 1, Ottawa, réf. du 25 février 2019, http:// publications.gc.ca/collections/Collection/J3-2-2002-2F.pdf 
MINISTÈRE DE LA JUSTICE DU QUÉBEC (2016). Plan stratégique 2015-2020, Québec, réf. du 25 février 2019, https://www.justice.gouv.qc.ca/fileadmin/user_upload/contenu/documents/ Fr_francais_/centredoc/publications/ministere/plans-strategiques/plan-strat1520.pdf

MINISTĖRE DE LA JUSTICE DU QUÉBEC (2004). Lintervention judiciaire en matière de protection de la jeunesse : constats, difficultés et pistes de solution, Québec, réf. du 25 février 2019, https:// www.justice.gouv.qc.ca/fileadmin/user_upload/contenu/documents/Fr_francais_/centredoc/ rapports/systeme-judiciaire/prot-jeun.pdf

MNISTÈRE DE LA SANTÉ ET DES SERVICES SOCIAUX (1997). Bilan d'implantation de la politique de santé mentale, Québec, réf. du 25 février 2019, http://publications.msss.gouv.qc.ca/ msss/fichiers/1996/96_737.pdf

OTERO, Marcelo (2016). «Traiter les intraitables : L'univers des autorisations judiciaires de soins à Montréal», Nouvelles pratiques sociales, Vol. 28, N² 2, p. 203-226.

OTERO, Marcelo (2007). «Le psychosocial dangereux, en danger et dérangeant : nouvelle figure des lignes de faille de la socialité contemporaine", Sociologie et sociétés, Vol. 39, No 1, p. 51-78.

OXFAM (2018). Partager la richesse avec celles et ceux qui la créent, Oxford, réf du 25 février 2019, https://reporterre.net/IMG/pdf/oxfam-rapport_ine_galite_s_2018.pdf

PARK, Lisa Sun-Hee (2011). «Criminalizing immigrant mothers : Public charge, health care, and welfare reform", International Journal of Sociology of the Family, Vol. 37, $\mathrm{N}^{\circ}$ 1, p. 27-47.

PIKETTY, Thomas (2013a). Le capital au XXI' siècle, Paris, Seuil, 976 p.

PIKETTY, Thomas (2013b). «La nouvelle prospérité des rentiers : la dynamique des inégalités dans un monde en croissance faible», dans Rémi GENEVEY et collab. (dirs.), Regards sur la Terre - Réduire les inégalités : un enjeu de développement durable, Paris, Armand Colin, p. 163-172.

PLEAU, Alexandre (2013). Les effets de la judiciarisation sur limplication parentale en protection de la jeunesse - Perception des intervenants, [mémoire de maitrise], Québec, Canada, Université Laval.

PROTECTEUR DU CITOYEN (2013). Rapport sur la contribution financière au placement des mineurs, Québec, réf. du 25 février 2019, https://protecteurducitoyen.qc.ca/sites/default/files/ pdf/rapports_speciaux/2013-03-21_contribution_financiere.pdf

Protection de la jeunesse - 072127, 2007 QCCQ 9540.

Protection de la jeunesse - 09430, 2009 QCCQ 10201.

Règlement sur l'aide juridique, c A-14, r. 2.

ROBERTSON, Michael, et Jeff GIDDINGS (2014). «Self-advocate in civil legal disputes : How personal and other factors influence the handing of their case", Melbourne University Law Review, Vol. 38, $\mathrm{N}^{\circ}$ 1, p. 119-150. 
SALLÉE, Nicolas, et Anaïs TSCHANZ (2018). “'C’est un peu la prison, mais c'est pas comme la vraie”. La carcéralité d'un centre de réadaptation pour jeunes délinquants à Montréal ", Métropolitiques, réf. du 18 octobre 2018, https://www.metropolitiques.eu/C-est-un-peu-la-prison-mais-c-est-pascomme-la-vraie.html.

SPADE, Dean (2015). Normal Life: Administrative violence, critical trans politics, and the limits of law, Durham, Duke University Press, 256 p.

VAYDA, Paul, et Stephen B. GINSBERG (2012). «Legal services plans : Crucial-time access to lawyers and the case for a public-private partnership», dans Michael TREBILCOCK, et collab. (dirs.), Middle Income Access to Justice, Toronto, University of Toronto Press, p. 246-268.

WACQUANT, Loïc (2010). "Crafting the neoliberal state: Workfare, prisonfare, and social insecurity», Sociological Forum, Vol. 25, No 2, p. 197-220.

WACQUANT, Loïc (1998). "Lıascension de lıÉtat pénal en Amérique», Actes de la recherche en sciences sociales, Vol. 124, p. 7-26.

WEXLER, David (1994) «An orientation to therapeutic jurisprudence», New England Journal on Criminal and Civil Confinement, Vol. 20, p. 259-264.

WILSON, James Q., et Georges KELLING (1982). «Broken windows - The police and neighborhood safety", The Atlantic Online, réf. du 10 octobre 2018, http://www.lakeclaire.org/docs/ BrokenWindows-AtlantaicMonthly-March82.pdf

WORD INEQUALITY LAB (2017). Rapport sur les inégalités mondiales 2018, réf. du 5 octobre 2018, https:/wir2018.wid.world/files/download/wir2018-summary-french.pdf

X (Dans la situation de), 2006 QCCQ 8425.

\section{Notes}

1 Dans cette réflexion, je désignerai par « droit social» l’ensemble des mécanismes juridiques destinés à soutenir la mission sociale de l'État.

2 Au Québec, les notaires peuvent également donner des conseils juridiques, mais comme ils ne représentent pas devant les tribunaux, ils sont exclus de cette réflexion. 